\title{
Factores asociados a la desnutrición o al riesgo de desnutrición en adultos mayores de San Juan de Pasto, Colombia: Un estudio transversal
}

\author{
Nubia Amparo Giraldo Giraldo ${ }^{a}$, Yenny Vicky Paredes Arturo ${ }^{b, *}$, Yelithza Idarraga Idarraga ${ }^{a}$, \\ Daniel Camilo Aguirre Acevedo ${ }^{b, c}$ \\ a $_{\text {Grup }}$ \\ b Grupo Desarrollo Humano y Social, Universidad Mariana, Colombia. \\ Crupo Académico de Epidemiología Clínica, Facultad de Medicina, Universidad de Antioquia, Colombia. \\ *yenny28_3@hotmail.com
}

Recibido el 19 de septiembre de 2016; aceptado el 8 de enero de 2017.

\section{PALABRAS CLAVE}

Desnutrición;

Factores de Riesgo;

Adulto Mayor.
Factores asociados a la desnutrición o al riesgo de desnutrición en adultos mayores de San Juan de Pasto, Colombia: Un estudio transversal

\section{RESUMEN}

Introducción: La desnutrición es un problema común en los adultos mayores relacionada tanto con los cambios propios del envejecimiento como con otros factores de salud, sociales y económicos. El objetivo de este estudio fue determinar la frecuencia de desnutrición o riesgo de desnutrición y los factores asociados, en adultos mayores de San Juan de Pasto, Nariño, Colombia.

Material y Métodos: Estudio transversal en una muestra de 261 adultos mayores. Para valorar el estado nutricional se utilizó el Mini Nutritional Assessment (MNA) y para la evaluación cognitiva se aplicó el Mini-Mental State Examination (MMSE). Los síntomas depresivos se determinaron a través de la escala Yesavage y el grado de independencia funcional con la escala de Lawton y Brody. Para establecer la asociación entre los factores sociodemográficos y clínicos con el MNA, se realizó un modelo de regresión logística y se calculó la Odds Ratio (OR) crudo y ajustado con su respectivo intervalo de confianza del 95\% (IC95\%).

Resultados: El 60,1\% de los adultos mayores presentó riesgo de desnutrición y el 6,5\% desnutrición. Quienes pertenecían a niveles socioeconómicos bajos (OR=2,07; IC95\%:1,18-3,64), con nivel de dependencia funcional (OR=2,74; IC95\%:1,07-5,53) y sintomatología depresiva (OR=7,02; IC95\%:2,08-23,74), presentaron mayor desnutrición o riesgo de desnutrición.

Conclusiones: Estos hallazgos muestran un alto porcentaje de adultos mayores con desnutrición o riesgo de desnutrición asociados a un nivel socioeconómico bajo, implicación o dependencia funcional y sintomatología depresiva. Se requiere un manejo integral orientado hacia la valoración e intervención planificada, teniendo en cuenta los factores asociados a esta condición nutricional. 
KEYWORDS

Malnutrition;

Risk Factors;

Aged.
Malnutrition or malnutrition risk related factors in older adults from San Juan de Pasto, Colombia: A cross sectional study

\section{ABSTRACT}

Introduction: Malnutrition is a common health problem in older adults; it is related to aging as with other health, social and economic factors. The aim of this study was to establish the frequency of malnutrition or malnutrition risk and associated factors in a sample of older adults located in San Juan de Pasto, Nariño, Colombia.

Material and Methods: This was a cross sectional study on a sample of 261 older adults. The Mini Nutritional Assessment (MNA) was used to rate the nutritional status. For cognitive evaluation, the Mini-Mental State Examination (MMSE) was used. Depressive symptoms were evaluated with Yesavage scale and the grade of functional independence with Lawton and Brody scale. A logistic regression model and raw and adjusted odds ratio (OR) with corresponding 95\% confidence interval were calculated to establish relation between clinic and sociodemographic factor with MNA.

Results: The $60.1 \%$ of older adults had malnutrition risk and $6.5 \%$ had malnutrition. Older adults who lived in low socioeconomic levels (OR=2,07; IC95\%: 1,18-3,64), with functional dependency (OR=2,74; IC95\%: 1,07-5,53) and had depressive symptoms (OR=7,02; IC95\%: 2,08-23,74) showed a greater malnutrition or malnutrition risk.

Conclusions: These findings show a high percentage of older adults with malnutrition or malnutrition risk linked to low socioeconomical levels, functional dependency and depressive symptoms. Integrated management towards the evaluation and planned interventions is required accounting the factors that trigger malnutrition.

\section{CITA}

Giraldo Giraldo NA, Paredes Arturo YV, Idarraga Idarraga Y, Aguirre Acevedo DC. Factores asociados a la desnutrición o al riesgo de desnutrición en adultos mayores de San Juan de Pasto, Colombia: Un estudio transversal. Rev Esp Nutr Hum Diet. 2017; 21(1): 39-48. doi: 10.14306/renhyd.21.1.288

\section{INTRODUCCIÓN}

El envejecimiento es un fenómeno que se viene presentando de forma más acelerada, en casi 30 años se proyecta en el mundo un incremento de la población adulta mayor (60 o más años de edad) de tres veces más ${ }^{1}$. En la región de las Américas, este grupo se duplicará y, aunque en la actualidad los países europeos cuentan con la mayor cantidad de población más vieja del mundo, las estadísticas prevén que el incremento de los adultos mayores ocurrirá de forma más rápida en los países en desarrollo, lo que constituye un hecho de transición demográfica, ligado a problemas relacionados con la salud, la alimentación y la nutrición².

La desnutrición puede entenderse como un déficit de energía, proteínas u otros nutrientes que afecta los tejidos y la composición corporal, generando efectos adversos en la funcionalidad e incrementando el riesgo de morbilidad y mortalidad ${ }^{3}$. Particularmente en los adultos mayores, la desnutrición es una condición multifactorial agravada por los cambios propios del envejecimiento como son la disminución de la masa magra, alteraciones fisiológicas del sistema digestivo, pérdida sensorial del olfato y el gusto, y pérdidas dentales acompañadas de la presencia de enfermedades crónicas, polifarmacia, depresión, problemas cognitivos y aislamiento social. Estos factores actúan de manera sinérgica haciendo que esta población sea más vulnerable a esta condición nutricional ${ }^{4}$.

La desnutrición en este grupo poblacional es elevada, siendo más alta en los hospitalizados (35-65\%), seguidos de los institucionalizados (30-60\%) y en menor proporción en los independientes $(5-10 \%)^{5}$. Para evaluar el estado nutricional de los adultos mayores se utilizan diversos métodos, 
sin embargo, el Mini Nutritional Assessment (MNA) es el más recomendado por ser simple y confiable, además ha sido validado en diferentes países Francia, México y Brasil, además, puede ser usado en quienes se encuentran hospitalizados, en cuidados en casa y en ambulatorios $6,7,8$.

El proceso de envejecimiento usualmente está acompañado de cambios psicológicos, fisiológicos, sociales y económicos, los cuales pueden afectar el estado nutricional del adulto mayor ${ }^{9}$. Enfermedades concomitantes ${ }^{10,11}$, síntomas depresivos ${ }^{12}$, alteración del estado cognitivo ${ }^{13}$ y dependencia funcional ${ }^{14}$ pueden incrementar el riesgo de desnutrición en esta población. Pocos estudios se han realizado en el contexto latinoamericano ${ }^{15,16}$ y colombiano ${ }^{17}$, donde la población adulta mayor presenta condiciones de vulnerabilidad y descuido por parte del estado ${ }^{18}$, que hace necesario conocer la frecuencia de desnutrición o riesgo de desnutrición y los factores asociados, con el propósito de revisar las políticas públicas actuales.

Por lo anterior, el objetivo principal de este estudio fue determinar los factores asociados a la desnutrición o riesgo de desnutrición en una muestra de adultos mayores de la ciudad San Juan de Pasto, Nariño, Colombia.

\section{MATERIAL Y MÉTODOS}

Estudio de corte transversal para analizar la relación de factores cognitivos, emocionales, clínicos y demográficos con desnutrición o riesgo de desnutrición en un grupo de adultos mayores. La fuente de información provino de los datos del estudio sobre caracterización de los factores multidimensionales de personas mayores de 60 años de la ciudad San Juan de Pasto realizado entre 2012 y $2014^{19}$. La población diana fueron adultos mayores de la ciudad San Juan de Pasto, capital del departamento de Nariño, Colombia. Esta ciudad se encuentra localizada en el suroeste de Colombia con una población aproximada de 500.000 habitantes, de los que cerca del $10 \%$ son adultos mayores. Los criterios de inclusión fueron: ser un adulto de 60 años o más, residir en la ciudad San Juan de Pasto, aceptar voluntariamente la participación en el estudio y firmar el consentimiento informado. Los criterios de exclusión fueron: no poder leer o escribir, dado que podían afectar el rendimiento en el instrumento de rastreo cognitivo o información incompleta en la escala MNA. En el estudio realizado entre 2012 y 2014 se incluyeron 426 adultos mayores, de estos se excluyeron 165 participantes, dado que tenían información incompleta en la escala $M N A^{8}$, o no podían leer o escribir lo que impedía la aplicación de las pruebas cognitivas. Los adultos mayores que participaron en la investigación fueron contactados a partir de miembros de su comunidad (barrios) de la ciudad de San Juan de Pasto. El proyecto siguió los principios éticos contenidos en la declaración del Helsinki de la Asociación Médica Mundial y contó con aprobación del comité de ética de la Universidad Mariana.

La evaluación fue realizada por profesionales de la salud del área de geriatría y enfermería, profesores de la Universidad Mariana de Pasto, quienes realizaron dicha evaluación en el domicilio del participante. El proceso de valoración nutricional se realizó utilizando el MNA versión completa ${ }^{8}$. EI MNA está compuesto por 18 ítems, con las siguientes valoraciones: general (movilidad, estilos de vida, y medicación), antropometría (peso, talla, circunferencia braquial y circunferencia de la pantorrilla), datos de alimentación (número de comidas, ingesta de alimentos y líquidos y autonomía para alimentarse) y valoración subjetiva (autopercepción de salud y nutrición). La presencia de desnutrición o riesgo de desnutrición se definió de la siguiente manera: desnutrición $<17$ puntos; riesgo de desnutrición entre 17 y 23,5 y buen estado nutricional $\geq 24$.

Se consideraron factores demográficos evaluados de acuerdo a un cuestionario prediseñado y según autorreporte del participante o algún familiar o cuidador responsable. Se evaluó la edad en años, el género (masculino y femenino), la escolaridad (si sabía leer/escribir, primaria, secundaria y estudios superiores), el estado civil (soltero, casado/unión libre, viudo y separado/divorciado), el estrato socioeconómico de acuerdo a la clasificación demográfica colombiana según el Departamento Administrativo Nacional de Estadísticas (clasifica a la población en seis niveles: 1 a 2: Bajo; 3 al 4: Medio; y 5 a 6: Alto), el estado de afiliación al Sistema General de Seguridad Social en Salud (clasifica a la población colombiana en tres regímenes: contributivo, subsidiado y beneficiario), la situación laboral (empleado/independiente, jubilado/ pensionado) y la dependencia económica (sí/no). Se evaluó el antecedente de enfermedades infecciosas, crónicas, respiratorias y músculo-esqueléticas a partir del autorreporte con un cuestionario prediseñado donde para cada uno se usó la siguiente instrucción: "A continuación voy a enumerarle una serie de enfermedades, ¿cuál de ellas ha sufrido o tiene actualmente?". Entre ellas se evaluó la presencia de hipertensión arterial (HTA), enfermedad cerebrovascular, diabetes, obesidad, enfermedad pulmonar obstructiva crónica (EPOC), neumonía, osteoporosis, artritis, cataratas, fracturas, cáncer y demencia.

La evaluación cognitiva se realizó utilizando el examen Mini-Mental State Examination (MMSE) ${ }^{20}$. La puntuación total máxima es de 30 y los principales parámetros de normalidad son la edad y la escolarización. En la práctica 
Factores asociados a la desnutrición o al riesgo de desnutrición en adultos mayores de San Juan de Pasto, Colombia: Un estudio transversal

diaria, una puntuación menor de 24 sugiere demencia, entre 23-21 demencia leve, entre 20-11 demencia moderada y menor de 10 una demencia severa. Los síntomas depresivos se evaluaron con la escala de depresión geriátrica de Yesavage ${ }^{21}$, teniendo en cuenta tres categorías de acuerdo al puntaje total obtenido a partir de la suma de los 15 ítems: de 0 a 5 normal, 6 a 10 depresión moderada y de 11 a 15 depresión severa. Se evaluó también el grado de independencia funcional con la escala de Lawton y Brody ${ }^{22}$, este instrumento valora 8 ítems (capacidad para utilizar el teléfono, hacer compras, preparación de la comida, cuidado de la casa, lavado de la ropa, uso de medios de transporte, responsabilidad respecto a la medicación y administración de su economía) y les asigna un valor numérico 1 (independiente) o 0 (dependiente). La puntuación final es la suma del valor de todas las respuestas y entre 0 (máxima dependencia) y 8 (independencia total).

Las características demográficas, la frecuencia de desnutrición o riesgo de desnutrición según la escala MNA, los antecedentes de enfermedades y la frecuencia de síntomas depresivos fueron descritas utilizando la frecuencia absoluta y relativa (\%) para las variables cualitativas. Se analizó la relación de los factores cognitivos, emocionales y demográficos con el riesgo de desnutrición categorizada de manera dicotómica como presencia de desnutrición + riesgo de desnutrición frente a ausencia (estado nutricional normal) según el MNA. En el análisis univaridado se utilizó la prueba $\chi^{2}$ dado que todas las variables fueron manejadas de manera categórica. Para cada factor se calculó el Odds Ratio (OR) crudo y ajustado con su respectivo intervalo de confianza del 95\% (IC95\%). Las variables que se tuvieron en cuenta para el cálculo del OR ajustado fueron ingresadas al modelo de regresión logística si cumplían con el criterio de Hosmer-Lemeshow (valor $p<0,25$ ) en el análisis univariado. Los análisis fueron realizados en el paquete estadístico IBM SPSS versión 21.0.

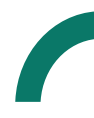

\section{RESULTADOS}

Características sociodemográficas y antecedentes médicos: El 60,9\% fue de género femenino, alrededor de un $50 \%$ se encontró en edades entre los 65 y 74 años, el 68\% alcanzó un nivel de escolaridad básica primaria, un $77 \%$ pertenecía al estrato socioeconómico bajo y un 59,8\% manifestó dependencia de otros familiares (Tabla 1 ).

Antecedentes médicos: Los principales antecedentes médicos (Tabla 2) reportados fueron en primer lugar la presencia de HTA $(55,2 \%)$, seguido por artritis $(36,8 \%)$, cataratas
$(31,4 \%)$, fracturas $(25,3 \%)$, osteoporosis $(21,1 \%)$, enfermedad cerebrovascular $(15,5 \%)$, obesidad $(17,6 \%)$ y diabetes mellitus tipo 2 (13,0\%). Otros antecedentes como EPOC, neumonía, cáncer y demencia tuvieron una frecuencia menor al 10\%. Un 14,2\% presentaron sintomatología depresiva (Yesavage $>5$ ) y un 32,6\% presentaron síntomas de deterioro cognitivo (MMSE<24).

Frecuencia de desnutrición y riesgo de desnutrición según características sociodemográficas y antecedentes médicos: En la muestra de estudio un $6,5 \%(n=17)$ y un $60,1 \%(n=157)$ presentó desnutrición o riesgo de desnutrición respectivamente. Las Tablas 1 y 2 presentan el estado nutricional evaluado con la escala MNA según las características sociodemográficas y clínicas de acuerdo a la desnutrición o riesgo de desnutrición. Se encontró una asociación estadística entre el riesgo de desnutrición y el estrato socioeconómico $(p=0,015)$, situación laboral $(p=0,006)$ y dependencia económica $(p=0,002)$. No se encontró asociación estadística ( $p>0,05)$ con los demás factores, aunque se presentó una mayor frecuencia de desnutrición o riesgo de desnutrición en adultos mayores de género femenino y de 80 o más años de edad. También se encontró asociación significativa con HTA, síntomas depresivos y con dependencia funcional. Los otros antecedentes médicos no se encontraron asociados estadísticamente.

Factores asociados a la desnutrición o riesgo de desnutrición según MNA: La Tabla 3 presenta la relación de los factores asociados con desnutrición o riesgo de desnutrición. Los resultados muestran que adultos mayores que pertenecen a niveles socioeconómicos bajos (OR=2,07; IC95\%:1,18-3,64), y con sintomatología depresiva severa (OR=7,02; IC95\%:2,08-23,74) presentan mayor riesgo de desnutrición. Comparado con los adultos mayores con independencia funcional, aquellos con funcionalidad afectada ligera (OR=1,92; IC95\%:1,03-3,58) y moderada (OR=2,89; IC95\%:1,14-7,31) aumentó casi dos a tres veces respectivamente las posibilidades de riesgo de desnutrición o desnutrición. Otros factores sociodemográficos o antecedentes médicos no se encontraron asociados estadísticamente.

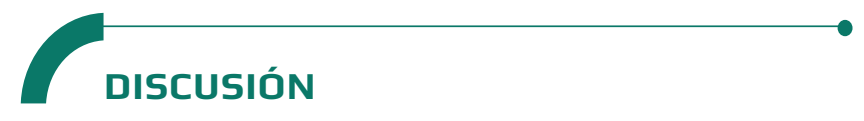

Un alto porcentaje de la población analizada se encuentra con desnutrición o en riesgo asociado con un nivel socioeconómico bajo, dependencia funcional y síntomas de depresión. Según estudios secundarios de los resultados de la Encuesta Multicéntrica de Salud, Bienestar y Envejecimiento en México y Brasil (SABE), las cifras de riesgo de 
Tabla 1. Estado nutricional según características sociodemográficas de 261 adultos mayores de la ciudad San Juan de Pasto, Colombia.

\begin{tabular}{|c|c|c|c|c|c|c|c|c|c|}
\hline & \multicolumn{2}{|c|}{$\begin{array}{l}\text { Desnutrición } \\
\qquad(n=17)\end{array}$} & \multicolumn{2}{|c|}{$\begin{array}{l}\text { Riesgo de } \\
\text { desnutrición } \\
(n=157)\end{array}$} & \multicolumn{2}{|c|}{ Normal $(n=87)$} & \multirow{2}{*}{$\begin{array}{c}\text { Muestra } \\
\text { total } \\
(n=261)\end{array}$} & \multirow[t]{2}{*}{$X^{2} ; g l$} & \multirow[t]{2}{*}{ Valor $\mathrm{p}$} \\
\hline & Frecuencia & Porcentaje & Frecuencia & Porcentaje & Frecuencia & Porcentaje & & & \\
\hline Género & & & & & & & & 4,$6 ; 2$ & 0,100 \\
\hline Femenino & 13 & 8,2 & 100 & 62,9 & 46 & 28,9 & 159 & & \\
\hline Masculino & 4 & 3,9 & 57 & 55,9 & 41 & 40,2 & 102 & & \\
\hline Edad en años & & & & & & & & 10,$5 ; 8$ & 0,232 \\
\hline $60-64$ & 2 & 4,2 & 29 & 60,4 & 17 & 35,4 & 48 & & \\
\hline $65-69$ & 5 & 7,0 & 47 & 66,2 & 19 & 26,8 & 71 & & \\
\hline $70-74$ & 3 & 4,9 & 36 & 59,0 & 22 & 36,1 & 61 & & \\
\hline $75-79$ & 2 & 5,3 & 27 & 71,1 & 9 & 23,7 & 38 & & \\
\hline 80 o más & 5 & 11,6 & 18 & 41,9 & 20 & 46,5 & 43 & & \\
\hline Escolaridad & & & & & & & & 5,$0 ; 6$ & 0,539 \\
\hline Sabe leer/escribir & 3 & 9,7 & 19 & 61,3 & 9 & 29,0 & 31 & & \\
\hline Primaria & 12 & 6,7 & 105 & 58,7 & 62 & 34,6 & 179 & & \\
\hline Secundaria & 2 & 5,0 & 28 & 70,0 & 10 & 25,0 & 40 & & \\
\hline Superiores & 0 & 0,0 & 5 & 45,5 & 6 & 54,5 & 11 & & \\
\hline Estrato socioeconómico & & & & & & & & 8,$4 ; 2$ & 0,015 \\
\hline Bajo & 16 & 7,9 & 127 & 62,9 & 59 & 29,2 & 202 & & \\
\hline Medio/Alto & 1 & 1,9 & 30 & 50,8 & 28 & 47,5 & 59 & & \\
\hline Estado de afiliación Salud & & & & & & & & 4,$6 ; 2$ & 0,100 \\
\hline Contribuyente & 0 & 0,0 & 28 & 59,6 & 19 & 40,4 & 47 & & \\
\hline Beneficiario/Subsidiado & 17 & 7,9 & 129 & 60,3 & 68 & 31,8 & 214 & & \\
\hline Estado Civil & & & & & & & & 3,$1 ; 4$ & 0,543 \\
\hline Soltero & 6 & 10,9 & 32 & 58,2 & 17 & 30,9 & 55 & & \\
\hline Casado/Unión libre & 8 & 6,2 & 76 & 58,5 & 46 & 35,4 & 130 & & \\
\hline Viudo/Separado/Divorciado & 3 & 5,2 & 49 & 84,5 & 24 & 41,4 & 58 & & \\
\hline Situación laboral & & & & & & & & 1,$3 ; 2$ & 0,006 \\
\hline Empleado/Independiente & 3 & 2,8 & 57 & 53,8 & 46 & 43,4 & 106 & & \\
\hline Jubilado/pensionado & 14 & 9,0 & 100 & 64,5 & 41 & 26,5 & 155 & & \\
\hline Tiene un cuidador & 13 & 5,9 & 134 & 60,4 & 75 & 33,8 & 222 & 1,$1 ; 2$ & 0,581 \\
\hline Dependencia económica & 15 & 9,6 & 100 & 64,1 & 41 & 26,3 & 156 & 12,$5 ; 2$ & 0,002 \\
\hline
\end{tabular}

$\mathbf{X}^{2}$ : Prueba Chi cuadrado; gl: grados de libertad.

desnutrición fueron $31,8 \%$ y 25,6\% respectivamente 23,24 , sin embargo, en la presente muestra evaluada por el MNA fue mayor (60,1\%). En Colombia, en un estudio realizado en las ciudades de Bogotá y Medellín, las dos principales capitales del país, la frecuencia de riesgo de desnutrición también fue más baja (34,3\% y $41 \%$ respectivamente) comparada con la encontrada en el presente estudio ${ }^{17,25}$. El hecho de que el riesgo de desnutrición o la presencia de desnutrición en los adultos mayores de Pasto sea superior a estos países o ciudades comparadas, lleva a pensar que las condiciones de seguridad alimentaria de la región y otros factores sociales podrían estar contribuyendo a esta problemática, lo que requiere de una mayor atención por parte de las autoridades y líderes de las políticas públicas en nuestra región.

En la ciudad de Medellín, casi la mitad de los adultos de 60 años y más se encontraban con desnutrición o en riesgo de 
Tabla 2. Estado nutricional según antecedentes médicos, nivel emocional, cognitivo y funcional de 261 adultos mayores de la ciudad San Juan de Pasto, Colombia.

\begin{tabular}{|c|c|c|c|c|c|c|c|c|c|}
\hline & \multicolumn{2}{|c|}{$\begin{array}{l}\text { Desnutrición } \\
(n=17)\end{array}$} & \multicolumn{2}{|c|}{$\begin{array}{c}\text { Riesgo de } \\
\text { desnutrición } \\
(n=157)\end{array}$} & \multicolumn{2}{|c|}{ Normal $(n=87)$} & \multirow{2}{*}{$\begin{array}{c}\text { Muestra } \\
\text { total } \\
(n=261)\end{array}$} & \multirow[t]{2}{*}{$X^{2} ; \mathrm{gl}$} & \multirow[t]{2}{*}{ Valor $p$} \\
\hline & Frecuencia & Porcentaje & Frecuencia & Porcentaje & Frecuencia & Porcentaje & & & \\
\hline \multicolumn{10}{|l|}{ Antecedentes médicos } \\
\hline HTA & 11 & 7,6 & 90 & 62,5 & 43 & 29,9 & 144 & 2,$1 ; 2$ & 0,353 \\
\hline Enfermedad cerebrovascular & 4 & 9,3 & 27 & 62,8 & 12 & 27,9 & 43 & 1,$1 ; 2$ & 0,569 \\
\hline Diabetes & 3 & 8,8 & 20 & 58,8 & 11 & 32,4 & 34 & 0,$3 ; 2$ & 0,842 \\
\hline Obesidad & 4 & 8,7 & 32 & 69,6 & 10 & 21,7 & 46 & 3,$5 ; 2$ & 0,175 \\
\hline EPOC & 1 & 7,7 & 11 & 84,6 & 1 & 7,7 & 13 & 4,$1 ; 2$ & 0,129 \\
\hline Neumonía & 3 & 14,3 & 13 & 61,9 & 5 & 23,8 & 21 & 2,$8 ; 5$ & 0,253 \\
\hline Osteoporosis & 5 & 9,1 & 37 & 67,3 & 13 & 23,6 & 55 & 3,$3 ; 2$ & 0,196 \\
\hline Artritis & 8 & 8,3 & 63 & 65,6 & 25 & 26,0 & 96 & 3,$9 ; 2$ & 0,139 \\
\hline Cataratas & 5 & 6,1 & 49 & 59,8 & 28 & 34,1 & 82 & 0,$6 ; 2$ & 0,971 \\
\hline Fracturas & 4 & 6,1 & 43 & 65,2 & 19 & 28,8 & 66 & 0,$9 ; 2$ & 0,624 \\
\hline Cáncer & 0 & 0,0 & 3 & 60,0 & 2 & 40,0 & 5 & 0,$4 ; 2$ & 0,819 \\
\hline Demencia & 4 & 19,0 & 15 & 71,4 & 2 & 9,5 & 21 & 9,$9 ; 2$ & 0,007 \\
\hline Síntomas depresivos & & & & & & & & 81,$5 ; 4$ & $<0,001$ \\
\hline Normal & 1 & 0,7 & 85 & 55,9 & 66 & 43,4 & 152 & & \\
\hline Leve (Yesavage 6-10) & 2 & 2,8 & 51 & 70,8 & 19 & 26,4 & 72 & & \\
\hline Severa (Yesavage $>10)$ & 14 & 37,8 & 21 & 56,8 & 2 & 5,4 & 37 & & \\
\hline MMSE $<24$ & 11 & 12,9 & 51 & 60,0 & 23 & 27,1 & 85 & 9,$5 ; 2$ & 0,009 \\
\hline $\begin{array}{l}\text { Independencia funcional } \\
\text { (Lawton y Brody) }\end{array}$ & & & & & & & & 17,$8 ; 2$ & $<0,001$ \\
\hline Dependencia & 11 & 17,7 & 36 & 58,1 & 15 & 24,2 & 62 & & \\
\hline Independencia & 6 & 3,0 & 121 & 60,8 & 72 & 36,2 & 199 & & \\
\hline
\end{tabular}

HTA: hipertensión arterial; EPOC: enfermedad pulmonar obstructiva crónica; MMSE: Mini-Mental State Examination; $\mathbf{X}^{2}$ : prueba Chi cuadrado; gl: grados de libertad.

desnutrición, con mayor frecuencia en mujeres, en edades más avanzadas, en los estratos más bajos, menor nivel educativo y en la zona rural ${ }^{25}$. En Pasto, esta situación fue similar, encontrando cifras más altas de desnutrición o riesgo de desnutrición en los de menor escolaridad y estrato socioeconómico bajo. Esto podría reflejar las inequidades sociales y de salud que existen en Colombia. En concordancia con lo anterior, otros autores reportan en sus estudios la asociación significativa entre el bajo nivel educativo y los bajos ingresos con la desnutrición ${ }^{26,27}$. Contrario a esto, estudios realizados en Líbano y España, concluyeron que el riesgo de desnutrición no estuvo asociado a la edad, el estado socioeconómico o el sexo ${ }^{28,29}$, pero en este último se utilizó la forma corta del MNA.
Respecto a las enfermedades crónicas, en este estudio se encontró que la HTA fue la más frecuente con un $55,2 \%$, situación similar se observó en Santiago de Chile y Sao Paulo según la encuesta SABE $2000^{30}$. En cuanto a la diabetes meIlitus tipo 2, en esta investigación la proporción fue de 13\%, resultado igual a lo reportado en la ciudad de Santiago de Chile y similar a Buenos Aires y Montevideo de acuerdo a la misma encuesta $\mathrm{SABE}^{30}$. La artritis, una enfermedad común e incapacitante, fue la segunda más frecuente en el presente estudio con un $36,8 \%$, pero menor al compararla con Buenos Aires, Montevideo y Bridgetown ${ }^{30}$. Las enfermedades pulmonares representaron un $13 \%$, datos iguales se reportaron en las ciudades de Santiago de Chile y Sao Paulo ${ }^{30}$. Estudios previos han mostrado asociación entre este tipo de 
Rev Esp Nutr Hum Diet. 2017; 21(1): 39 - 48

Factores asociados a la desnutrición o al riesgo de desnutrición en adultos mayores de San Juan de Pasto, Colombia: Un estudio transversal

Tabla 3 . Factores sociodemográficos, clínicos asociados con riesgo de desnutrición o desnutrición en adultos mayores de la ciudad San Juan de Pasto, Nariño, Colombia.

\begin{tabular}{|c|c|c|c|c|}
\hline & OR Crudo & (IC95\%) & OR ajustado & (IC95\%) \\
\hline \multicolumn{5}{|l|}{ Género } \\
\hline $\begin{array}{l}\text { Femenino } \\
\text { Masculino }\end{array}$ & $\begin{array}{c}1,65 \\
\text { Referencia }\end{array}$ & $(0,98-2,79)$ & $\begin{array}{c}1,54 \\
\text { Referencia }\end{array}$ & $(0,86-2,74)$ \\
\hline \multicolumn{5}{|l|}{ Edad en años } \\
\hline $60-64$ & Referencia & & Referencia & \\
\hline $65-69$ & 1,50 & $(0,68-3,31)$ & 1,73 & $(0,77-3,89)$ \\
\hline $70-74$ & 0,97 & $(0,44-2,14)$ & 1,03 & $(0,45-2,32)$ \\
\hline $75-79$ & 1,77 & $(0,68-4,59)$ & 1,25 & $(0,50-3,17)$ \\
\hline 80 o más & 0,63 & $(0,27-1,46)$ & 0,65 & $(0,26-1,60)$ \\
\hline \multicolumn{5}{|l|}{ Escolaridad } \\
\hline Sabe leer/escribir & 2,93 & $(0,71-12,11)$ & 2,35 & $(0,58-9,60)$ \\
\hline Primaria & 2,26 & $(0,66-7,72)$ & 1,74 & $(0,47-6,41)$ \\
\hline Secundaria & 3,60 & $(0,90-14,39)$ & 2,90 & $(0,68-12,27)$ \\
\hline Superiores & Referencia & & Referencia & \\
\hline \multicolumn{5}{|l|}{ Estrato socioeconómico } \\
\hline Medio/Alto & Referencia & & Referencia & \\
\hline Bajo & 2,24 & $(1,27-3,95)$ & 2,07 & $(1,18-3,64)$ \\
\hline \multicolumn{5}{|l|}{ Estado de afiliación Salud } \\
\hline Contribuyente & Referencia & & Referencia & \\
\hline Beneficiario & 0,85 & $(0,38-1,87)$ & 0,55 & $(0,22-1,36)$ \\
\hline Subsidiado & 1,79 & $(0,91-3,52)$ & 0,95 & $(0,43-2,12)$ \\
\hline \multicolumn{5}{|l|}{ Estado Civil } \\
\hline Casado/Unión libre & Referencia & & Referencia & \\
\hline Soltero & 1,22 & $(0,62-2,41)$ & 1,10 & $(0,54-2,23)$ \\
\hline Viudo & 1,22 & $(0,63-2,36)$ & 1,11 & $(0,55-2,21)$ \\
\hline Separado/divorciado & 1,10 & $(0,39-3,11)$ & 1,17 & $(0,40-3,45)$ \\
\hline \multicolumn{5}{|l|}{ Situación laboral } \\
\hline Empleado & Referencia & & Referencia & \\
\hline Independiente & 0,67 & $(0,30-1,50)$ & 0,72 & $(0,30-1,71)$ \\
\hline Jubilado/pensionado & 1,85 & $(1,02-3,37)$ & 1,57 & $(0,83-2,97)$ \\
\hline Dependencia económica & 2,19 & $(1,29-3,70)$ & 1,64 & $(0,95-2,80)$ \\
\hline Independiente económicamente & Referencia & & Referencia & \\
\hline Tiene un cuidador a cargo & 0,87 & $(0,42-1,81)$ & 0,64 & $(0,30-1,35)$ \\
\hline \multicolumn{5}{|l|}{ Antecedentes médicos } \\
\hline HTA & 1,42 & $(0,84-2,37)$ & 1,19 & $(0,69-2,06)$ \\
\hline Enfermedad cardiovascular & 1,35 & $(0,66-2,79)$ & 0,71 & $(0,18-2,77)$ \\
\hline Enfermedad cerebrovascular & 1,89 & $(0,51-6,96)$ & 1,35 & $(0,61-3,00)$ \\
\hline Diabetes & 1,05 & $(0,49-2,27)$ & 0,94 & $(0,41-2,16)$ \\
\hline EPOC & 6,37 & $(0,82-49,82)$ & 4,60 & $(0,98-21,68)$ \\
\hline Osteoporosis & 1,81 & $(0,91-3,59)$ & 1,60 & $(0,81-3,18)$ \\
\hline Cataratas & 0,95 & $(0,55-1,65)$ & 0,91 & $(0,51-1,60)$ \\
\hline Neumonía & 1,66 & $(0,59-4,69)$ & 0,94 & $(0,35-2,49)$ \\
\hline Artritis & 1,71 & $(0,98-2,98)$ & 1,28 & $(0,71-2,32)$ \\
\hline Fracturas & 1,32 & $(0,72-2,43)$ & 1,03 & $(0,57-1,88)$ \\
\hline Cáncer & 0,75 & $(0,12-4,55)$ & 1,10 & $(0,19-6,32)$ \\
\hline Demencia & 5,21 & $(1,18-22,91)$ & 2,75 & $(0,72-10,53)$ \\
\hline Síntomas depresivos (Severa) (Yesavage $>10$ ) & 10,70 & $(2,51-45,63)$ & 7,02 & $(2,08-23,74)$ \\
\hline MMSE $<24$ & 1,54 & $(0,87-2,72)$ & 1,36 & $(0,75-2,46)$ \\
\hline \multicolumn{5}{|l|}{ Independencia funcional (Lawton y Brody) } \\
\hline Dependencia & 1,78 & $(0,93-3,4)$ & 2,74 & $(1,07-5,53)$ \\
\hline Independencia & Referencia & & Referencia & \\
\hline
\end{tabular}

HTA: hipertensión arterial; EPOC: enfermedad pulmonar obstructiva crónica; MMSE: Mini-Mental State Examination;

OR: Odds ratio; IC95\%: intervalo de confianza del 95\%; OR ajustado: por sexo, edad, estrato socioeconómico, afiliación al sistema de salud, dependencia económica, EPOC, obesidad, osteoporosis, artritis, síntomas depresivos (Yesavage) y dependencia funcional (Lawton y Brody). 
Factores asociados a la desnutrición o al riesgo de desnutrición en adultos mayores de San Juan de Pasto, Colombia: Un estudio transversal

enfermedades y el riesgo de desnutrición. Un estudio realizado en los Países Bajos donde también se utilizó el MNA para valorar el estado nutricional de los adultos mayores, se encontró que la desnutrición fue más prevalente y se asoció significativamente con la presencia de osteoporosis ${ }^{31}$. En dicho estudio se encontró también una mayor prevalencia de desnutrición en adultos mayores con enfermedad cerebrovascular y EPOC ${ }^{31}$. Una posible explicación para esto es que tanto la osteoporosis, la enfermedad cerebrovascular y la EPOC son más incapacitantes y limitan la movilidad llevando a la postración y a la desnutrición.

Asimismo, otras comorbilidades, como la presencia de depresión, se han asociado con el riesgo de desnutrición ${ }^{31}$. En el presente estudio se encontró que entre los adultos con presencia de desnutrición la mayoría tenían sintomatología depresiva. Una posible relación inversa puede explicar los resultados, donde un estado nutricional inadecuado, específicamente en la etapa de vejez, incrementa la vulnerabilidad ante enfermedades mentales como la depresión ${ }^{32}$. En este sentido, la literatura refiere que la presencia de riesgo nutricional en estadios avanzados de edad desencadena mayor sintomatología depresiva ${ }^{33}$. Una de las explicaciones se podría atribuir a la deficiencia de algunos nutrientes como vitaminas y minerales, los cuales intervienen en el funcionamiento cerebral a nivel de la transmisión simpática ${ }^{34}$. Por tanto, las personas con una ingesta deficiente de estos nutrientes, tienden a presentar más alteraciones de orden emocional. De esta manera, la depresión puede desencadenarse por factores dietéticos, donde el estado nutricional desempeñaría un papel relevante en la prevención de un cuadro depresivo, y a su vez, la sintomatología depresiva puede modificar los hábitos alimentarios y alterar la situación nutricional del paciente ${ }^{35}$. Por tanto, la depresión y desnutrición son trastornos geriátricos comunes con una connotación multifactorial que puede resultar en un círculo vicioso que afectaría al adulto mayor por largo tiempo.

En otro contexto, pese a que el ámbito político, demográfico y socioeconómico varía de un país a otro e incluso de una ciudad a otra, la similitudes encontradas en la frecuencia de estas enfermedades crónicas pone de manifiesto que el grupo de adultos mayores por los cambios propios del envejecimiento como aumento de la grasa visceral, disminución de la masa muscular y alteraciones metabólicas, acompañadas de inactividad física, predisponen a dichas enfermedades crónicas que algunas veces se acompañan de desnutrición, como por ejemplo en la EPOC, que se asocia con incremento del metabolismo muscular respiratorio, inflamación sistémica y una limitada ingesta energética ${ }^{36}$.

En relación con las condiciones de mayor complejidad que acompañan el proceso de envejecimiento, la deficiencia nutricional, puede desencadenar consecuencias a nivel de la capacidad funcional en el adulto mayor ${ }^{37}$. En este sentido, varios autores refieren que el riesgo de desnutrición conlleva a una mayor dependencia en las actividades de la vida diaria en población senil ${ }^{38}$. Estos hallazgos concuerdan con lo encontrado en el presente estudio, presentándose mayor dependencia funcional en aquellos adultos mayores con riesgo de desnutrición. Al respecto, la literatura menciona que la desnutrición puede conducir al deterioro en las actividades de la vida diaria por la alteración en la capacidad funcional y, aunque la disminución de estas podría tener múltiples etiologías, el estado nutricional influye de manera relevante respecto a la prevalencia de limitaciones funcionales en este grupo etario en particular. Por tanto, la evaluación del estado nutricional en el adulto mayor es una prioridad, dado que está directamente relacionada con la capacidad del desempeño en las actividades de la vida cotidiana ${ }^{39}$.

Por otro lado, el creciente interés por establecer relaciones entre envejecimiento, nutrición y cognición, se ha constituido en un tema prioritario. Sin embargo, son muchos los factores que determinarían este tipo de explicaciones, entre ellos se encuentran variables como la edad, donde la investigación se ha focalizado en la vejez tardía, pero no se ha establecido si estas asociaciones están presentes únicamente en la edad avanzada o en otros estadios del ciclo evolutivo ${ }^{40}$. En este estudio no se pudo demostrar una asociación del nivel nutricional con el nivel cognitivo. Una explicación puede ser que la escala MNA presenta menor sensibilidad y especificidad en pacientes geriátricos con deterioro cognitivo. Dicho planteamiento se argumenta en el carácter subjetivo de la escala, sobre todo por el sesgo a nivel de la autopercepción del estado de salud que refiere el adulto mayor. Asimismo, la literatura reporta que una gran proporción de estos estudios se han focalizado en analizar aspectos más específicos del estado nutricional, como son la calidad y cantidad de nutrientes que este grupo poblacional consume ${ }^{41}$.

La principal limitación de este estudio es el carácter transversal que no permite responder a preguntas de tipo causal para la comprensión de los diferentes factores que pueden influir en el estado nutricional del adulto mayor. La relación entre los diferentes factores y el estado nutricional es más compleja que en otros grupos de edad debido a las múltiples alteraciones asociadas con el envejecimiento. De igual manera, algunas situaciones en el anciano son fluctuantes, especialmente aquellas que se relacionan con el estado cognitivo y emocional; sumado a ello el estado mental y funcional del anciano son elementos que influyen significativamente en la percepción de enfermedad o salud. Por tanto, no es fácil establecer relaciones donde la etiología se explica a partir de diversos agentes de orden biológico y psicosocial 
propios del ser humano. Asimismo, se debe tener presente que algunas variables se evaluaron a través de autorreporte, como los antecedentes médicos, lo que podría sugerir un sesgo de información. Otra limitación puede estar relacionada con la poca potencia estadística necesaria para establecer relaciones entre el estado nutricional y otros factores reportados por otros autores. Finalmente, la frecuencia del riesgo de desnutrición o desnutrición y el efecto de los factores aquí estudiados fueron estimados a partir de una muestra por conveniencia, lo que puede limitar las posibilidades de generalización de los resultados.

Este estudio es el primero que se realiza en la región sur colombiana a partir de un enfoque multidisciplinar para un abordaje integral del ser humano, sobre todo en esta etapa evolutiva, cuya característica básica es la pluralidad de factores que predisponen a la prevalencia de numerosas enfermedades acompañadas de desnutrición. Por otro lado, son pocos los estudios que evalúan el estado nutricional de los adultos mayores que viven en su contexto domiciliario. Normalmente, los trabajos de investigación se focalizan en grupos poblacionales institucionalizados.

Una fortaleza de esta investigación es el enfoque de evaluación multidimensional y del funcionamiento recomendado por la Organización Mundial de la Salud, orientado a la detección y explicación de problemas médicos, mentales, funcionales y sociales del adulto mayor. Por tanto, llevar a cabo este tipo de evaluación depende del trabajo coordinado e integrado de diferentes disciplinas, que desde sus procesos de intervención se focalizan en aspectos específicos de la valoración integral al adulto mayor.

El presente estudio proporciona información relevante sobre el estado nutricional y factores relacionados en población adulta mayor de la ciudad San Juan de Pasto. Estos hallazgos pueden orientar el diseño de estrategias y planificar intervenciones apropiadas con el fin de mejorar la calidad de vida y fomentar un envejecimiento exitoso. La desnutrición en población adulta mayor, se debe abordar como un síndrome geriátrico multifactorial, la cual requiere un manejo integral orientada hacia la valoración e intervención planificada, teniendo en cuenta los factores subyacentes que la desencadenan.

\section{$\longrightarrow$ \\ CONCLUSIONES}

En los adultos mayores de la ciudad San Juan de Pasto, Colombia, el riesgo de desnutrición o la desnutrición es muy frecuente. Un nivel socioeconómico bajo, dependencia funcional y síntomas de depresión sugieren una mayor asociación con esta condición nutricional en dicha población.

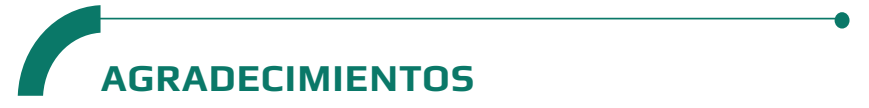

Los autores expresan su sincero agradecimiento a los adultos mayores de la ciudad San Juan de Pasto, Nariño, CoIombia, y al equipo de trabajo de campo y a la Universidad Mariana por su apoyo para la realización de este proyecto.

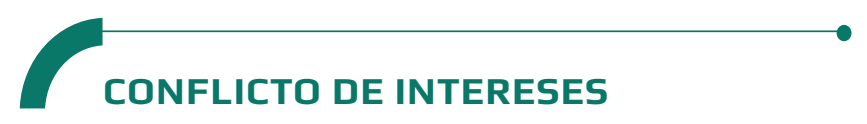

Los autores expresan que no hay conflictos de interés al redactar el manuscrito.

\section{$\longrightarrow$ REFERENCIAS}

(1) Naciones Unidas. Informe de la Segunda Asamblea Mundial sobre el Envejecimiento: Madrid, 8 a 12 de abril de 2002. Nueva York: Naciones Unidas; 2002.

(2) Tucker KL, Buranapin S. Nutrition and aging in developing countries. J Nutr. 2001; 131(9): 2417S-23S.

(3) Lochs H, Allison SP, Meier R, Pirlich M, Kondrup J, Schneider $S$, et al. Introductory to the ESPEN Guidelines on Enteral Nutrition: Terminology, definitions and general topics. Clin Nutr. 2006; 25(2): 180-6.

(4) Guyonnet S, Rolland Y. Screening for Malnutrition in Older People. Clin Geriatr Med. 2015; 31(3): 429-37.

(5) Brownie S. Why are elderly individuals at risk of nutritional deficiency? Int ] Nurs Pract. 2006; 12(2): 110-8.

(6) Hudgens J, Langkamp-Henken B. The Mini Nutritional Assessment as an assessment tool in elders in long-term care. Nutr Clin Pract. 2004; 19(5): 463-70.

(7) Machado RSP, Coelho MASC, Veras RP. Validity of the portuguese version of the mini nutritional assessment in brazilian elderly. BMC Geriatr. 2015; 15: 132.

(8) Guigoz Y, Vellas B, Garry PJ. Assessing the nutritional status of the elderly: The Mini Nutritional Assessment as part of the geriatric evaluation. Nutr Rev. 1996; 54(1 Pt 2): S59-65.

(9) Nykänen I, Lönnroos E, Kautiainen H, Sulkava R, Hartikainen $\mathrm{S}$. Nutritional screening in a population-based cohort of community-dwelling older people. Eur ] Public Health. 2013; 23(3): 405-9.

(10) Verbrugghe M, Beeckman D, Van Hecke A, Vanderwee K, Van Herck K, Clays E, et al. Malnutrition and associated factors in nursing home residents: a cross-sectional, multi-centre study. Clin Nutr. 2013; 32(3): 438-43.

(11) Ülger Z, Halil M, Kalan I, Yavuz BB, Cankurtaran M, Güngör $E$, et al. Comprehensive assessment of malnutrition risk and related factors in a large group of community-dwelling older adults. Clin Nutr. 2010; 29(4): 507-11. 
Factores asociados a la desnutrición o al riesgo de desnutrición en adultos mayores de San Juan de Pasto, Colombia: Un estudio transversal

(12) Peng L-N, Cheng Y, Chen L-K, Tung H-H, Chu K-H, Liang S-Y. Cognition and social-physiological factors associated with malnutrition in hospitalized older adults in Taiwan. J Nurs Res. 2015; 23(1): 1-5.

(13) El Zoghbi M, Boulos C, Amal AH, Saleh N, Awada S, Rachidi S, et al. Association between cognitive function and nutritional status in elderly: A cross-sectional study in three institutions of Beirut-Lebanon. Geriatr Ment Health Care. 2013; 1(4): 73-81.

(14) Serrano-Urrea R, García-Meseguer MJ. Relationships between nutritional screening and functional impairment in institutionalized Spanish older people. Maturitas. 2014; 78(4): 323-8.

(15) Contreras AL, Mayo GVA, Romaní DA, Tejada GS, Yeh M, Ortiz $\mathrm{PJ}$, et al. Malnutrición del adulto mayor y factores asociados en el distrito de Masma Chicche, Junín, Perú. Rev Med Hered. 2013; 24(3): 186-91.

(16) Rodríguez-Tadeo A, Wall-Medrano A, Gaytan-Vidaña ME, Campos A, Ornelas-Contreras M, Novelo-Huerta HI. Malnutrition risk factors among the elderly from the USMexico border: the "one thousand» study. J Nutr Health Aging. 2012; 16(5): 426-31.

(17) Chavarro-Carvajal D, Reyes-Ortiz C, Samper-Ternent R, Arciniegas AJ, Gutierrez CC. Nutritional assessment and factors associated to malnutrition in older adults: a cross-sectional study in Bogotá, Colombia. J Aging Health. 2015; 27(2): 304-19.

(18) Pelcastre-Villafuerte BE, Treviño-Siller S, González-Vázquez T, Márquez-Serrano M. Apoyo social y condiciones de vida de adultos mayores que viven en la pobreza urbana en México. Cad Saúde Pública. 2011; 27(3): 460-70.

(19) Rosas GM, Paredes YV, Yarce E, Rosero M, Morales A. Caracterización de los factores multidimensionales de las personas mayores de 60 años de la ciudad de San Juan de Pasto. Pasto, Colombia: Editorial UNIMAR; 2015.

(20) Folstein MF, Folstein SE, McHugh PR. «Mini-mental state». A practical method for grading the cognitive state of patients for the clinician. J Psychiatr Res. 1975; 12(3): 189-98.

(21) Aguirre-Acevedo DC, Gómez RD, Moreno S, Henao-Arboleda E, Motta M, Muñoz C, et al. Validez y fiabilidad de la batería neuropsicologica CERAD-Col. Rev Neurol. 2007; 45(11): 655-60.

(22) Lawton M, Brody E. Instrumental Activities of Daily Living (IADL) Scale. Original observer-rated version. «Does do» form-for women only. Psychopharmacol Bull. 1988; 24(4): 785-7.

(23) Franco-Álvarez N, Ávila-Funes JA, Ruiz-Arreguí L, GutiérrezRobledo LM. Determinantes del riesgo de desnutrición en los adultos mayores de la comunidad: análisis secundario del estudio Salud, Bienestar y Envejecimiento (SABE) en México. Rev Panam Salud Publica. 2007; 22(6): 369-75.

(24) Ferreira LS, do Amaral TF, Marucci M de FN, Nascimento LFC, Lebrão ML, Duarte YA de O. Undernutrition as a major risk factor for death among older Brazilian adults in the community-dwelling setting: SABE survey. Nutrition. 2011; 27(10): 1017-22.

(25) Giraldo NA, Deossa GC, Estrada A. Estado nutricional de los adultos mayores de los hogares de Medellín y sus corregimientos. En: Hoyos GM, editor. Perfil de Seguridad Alimentaria y Nutricional de Medellín y sus corregimientos, 2015. Medellín, Colombia: Alcaldía de Medellín; 2015. p. 343-77.

(26) Donini LM, Scardella P, Piombo L, Neri B, Asprino R, Proietti AR, et al. Malnutrition in elderly: social and economic determinants. J Nutr Health Aging. 2013; 17(1): 9-15.

(27) Timpini A, Facchi E, Cossi S, Ghisla MK, Romanelli G, Marengoni A. Self-reported socio-economic status, social, physical and leisure activities and risk for malnutrition in late life: a crosssectional population-based study. J Nutr Health Aging. 2011; 15(3): 233-8.

(28) Maseda A, Gómez-Caamaño S, Lorenzo-López L, LópezLópez R, Diego-Diez C, Sanluís-Martínez V, et al. Health determinants of nutritional status in community-dwelling older population: the VERISAÚDE study. Public Health Nutr. 2016; 19(12): 2220-8.

(29) Mitri R, Boulos C, Adib SM. Determinants of the nutritional status of older adults in urban Lebanon. Geriatr Gerontol Int. 2016.

(30) Peláez M, Palloni A, Albala C, Alfonso JC, Ham-Chande R, Hennis A, et al. SABE - Survey on Health, Well-Being, and Aging in Latin America and the Caribbean, 2000. ICPSR03546-v1 [Internet]. Ann Arbor, Ml: Inter-university Consortium for Political and Social Research; 2005. Disponible en: http://doi. org/10.3886/ICPSR03546.v1

(31) van Bokhorst-de van der Schueren MAE, Lonterman-Monasch $\mathrm{S}$, de Vries OJ, Danner SA, Kramer MHH, Muller M. Prevalence and determinants for malnutrition in geriatric outpatients. Clin Nutr. 2013; 32(6): 1007-11.

(32) Jung KI, Ock SM, Chung JH, Song CH. Associations of serum $\mathrm{Ca}$ and $\mathrm{Mg}$ levels with mental health in adult women without psychiatric disorders. Biol Trace Elem Res. 2010; 133(2): 153-61.

(33) Pérez E, Lizárraga DC, Martínez M del R. Asociación entre desnutrición y depresión en el adulto mayor. Nutr Hosp. 2014; 29(4): 901-6.

(34) Nazemi L, Skoog I, Karlsson I, Hosseini S, Hosseini M, Hosseinzadeh MJ, et al. Depression, prevalence and some risk factors in elderly nursing homes in tehran, iran. Iran ] Public Health. 2013; 42(6): 559-69.

(35) Vafaei Z, Mokhtari H, Sadooghi Z, Meamar R, Chitsaz A, Moeini $M$. Malnutrition is associated with depression in rural elderly population. J Res Med Sci. 2013; 18(Suppl 1): S15-19.

(36) Fuchs V, Sandoval J. Soporte nutricional en el paciente neumópata. Neumol Cir Torax. 2005; 64(1): 5-8.

(37) lizaka S, Tadaka E, Sanada H. Comprehensive assessment of nutritional status and associated factors in the healthy, community-dwelling elderly. Geriatr Gerontol Int. 2008; 8(1): 24-31.

(38) Boulos C, Salameh P, Barberger-Gateau P. The AMEL study, a cross sectional population-based survey on aging and malnutrition in 1200 elderly Lebanese living in rural settings: protocol and sample characteristics. BMC Public Health. 2013; 13: 573.

(39) Boscatto EC, Duarte M de F da S, Coqueiro R da S, Barbosa AR. Nutritional status in the oldest elderly and associated factors. Rev Assoc Med Bras. 2013; 59(1): 40-7.

(40) Tarazona FJ, Belenguer A, Doménech JR, Gac H, Cuesta $D$, Medina $L$, et al. Validez de la escala MNA como factor de riesgo nutricional en pacientes geriátricos institucionalizados con deterioro cognitivo moderado y severo. Nutr Hosp. 2009; 24(6): 724-31.

(41) Dosil A, Dosil C, Leal C, Neto S. Estado nutricional de ancianos con deterioro cognitivo. INFAD. 2013; 2(1): 297-310. 